\title{
Phonon deformation potentials in wurtzite GaN and ZnO determined by uniaxial pressure dependent Raman measurements
}

Cite as: Appl. Phys. Lett. 98, 061906 (2011); https://doi.org/10.1063/1.3554434

Submitted: 18 November 2010 . Accepted: 22 January 2011 . Published Online: 09 February 2011

G. Callsen, J. S. Reparaz, M. R. Wagner, R. Kirste, C. Nenstiel, A. Hoffmann, and M. R. Phillips

\section{ARTICLES YOU MAY BE INTERESTED IN}

Structural and optical investigation of non-polar (1-100) GaN grown by the ammonothermal method

Journal of Applied Physics 113, 203513 (2013); https://doi.org/10.1063/1.4807581

Raman and photoluminescence studies of biaxial strain in GaN epitaxial layers grown on $6 \mathrm{H}-\mathrm{SiC}$

Journal of Applied Physics 82, 5097 (1997); https://doi.org/10.1063/1.366310

Phonon deformation potentials of $\mathrm{a}-\mathrm{GaN}$ and $-\mathrm{AIN}$ : An ab initio calculation

Applied Physics Letters 77, 346 (2000); https://doi.org/10.1063/1.127009

Lock-in Amplifiers Find out more today

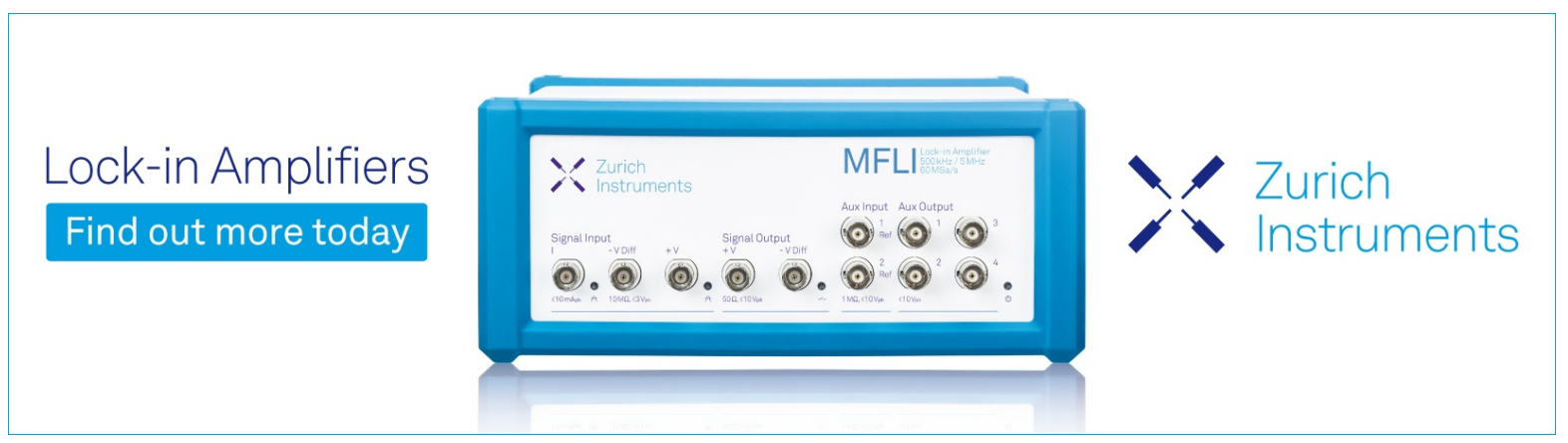

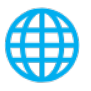

View Online 


\title{
Phonon deformation potentials in wurtzite GaN and ZnO determined by uniaxial pressure dependent Raman measurements
}

\author{
G. Callsen, ${ }^{1,2, a)}$ J. S. Reparaz, ${ }^{1}$ M. R. Wagner, ${ }^{1}$ R. Kirste, ${ }^{1}$ C. Nenstiel, ${ }^{1,2}$ A. Hoffmann, ${ }^{1}$ \\ and M. R. Phillips ${ }^{2}$ \\ ${ }_{1}^{1}$ Institut für Festkörperphysik, Technische Universität Berlin, Hardenbergstr. 36, 10623 Berlin, Germany \\ ${ }^{2}$ Department of Physics and Advanced Materials, University of Technology Sydney, P.O. Box 123, \\ Broadway, New South Wales 2007, Australia
}

(Received 18 November 2010; accepted 22 January 2011; published online 9 February 2011)

\begin{abstract}
We report the phonon deformation potentials of wurtzite $\mathrm{GaN}$ and $\mathrm{ZnO}$ for all zone center optical phonon modes determined by Raman measurements as a function of uniaxial pressure. Despite all the structural and optical similarities between these two material systems, the pressure dependency of their vibrational spectra exhibits fundamental distinctions, which is attributed to their different bond ionicities. In addition, the LO-TO splitting of the $A_{1}$ and $E_{1}$ phonon modes is analyzed which yields insight into the uniaxial pressure dependency of Born's transverse effective charge $e_{T}^{*}$. (C) 2011 American Institute of Physics. [doi:10.1063/1.3554434]
\end{abstract}

$\mathrm{GaN}$ and $\mathrm{ZnO}$ are the leading contenders for future lightemitting diode and laser diode technologies. Both materials are noted for their outstanding optical properties as well as the challenges involved in their growth, defect control, and doping. Homoepitaxial growth of both material systems seems to be an effective solution for overcoming these fabrication issues but has so far been proven too costly. Therefore, growth of $\mathrm{GaN}$ and $\mathrm{ZnO}$ on suitable alternative substrates has been undertaken. As a result, strain due to lattice mismatch has become an important variable in the epitaxial growth process of $\mathrm{GaN}$ and $\mathrm{ZnO}$ and explains why there is intense interest in tailoring the variable strain in the next generation of devices based on these materials. Evaluation of strain levels and their distribution in semiconductors is also pivotal to understanding fabrication of low dimensional structures involving strain induced growth mechanisms. Raman spectroscopy is the standard nondestructive method for the determination of the strain in bulk and nanostructured materials. This experimental technique relies on the precise knowledge of phonon deformation potentials (PDPs) which in turn allows the determination of the strain levels from the phonon frequencies. Although the optical properties of GaN and $\mathrm{ZnO}$ are well understood there is still a lack of knowledge on their basic lattice dynamic properties, in particular the phonon pressure coefficients and PDPs. Published experimental values ${ }^{1,2}$ in GaN to date are based on inhomogeneously biaxially strained layers and results from hydrostatic pressure dependent Raman measurements on bulk material ${ }^{3}$ which introduces significant errors in the determination of the PDPs. In the case of $\mathrm{ZnO}$ only the phonon deformation potentials of the nonpolar $E_{2}^{\text {high }}$ mode were reported using a comparable experimental approach. ${ }^{4}$ Therefore, the lack of sufficient corresponding experimental data for $\mathrm{GaN}$ and $\mathrm{ZnO}$ in the literature explains the limited adaptability of Raman spectroscopy for strain tailoring applications. This situation itself constitutes a strong motivation for the present work.

In this letter, we supply an overview for all optical first order Raman phonon mode pressure coefficients of $\mathrm{GaN}$ and $\mathrm{ZnO}$ and for the resulting PDPs. Raman spectra were recorded under the influence of compressive stress along the

${ }^{a)}$ Electronic mail: callsen@tu-berlin.de. c-axis of the crystals which combined with data for the hydrostatic pressure coefficients ${ }^{5,6}$ allowed the determination of the PDPs. Following the measurement of the phonon pressure coefficients for all LO and TO modes we are able to analyze the uniaxial pressure dependencies of the LO-TO splittings and related Born's transverse effective charge $e_{T}^{*}$. This analysis reveals a partly opposing behavior for $\mathrm{GaN}$ and $\mathrm{ZnO}$, which provides an insight into the different ionicities of the bonds.

The samples analyzed in this work were a c-plane GaN substrate grown by hydride vapor phase epitaxy and a hydrothermally grown $\mathrm{ZnO}$ c-plane substrate. Both crystals were cut into square shaped plates with a surface area of $\approx 4 \mathrm{~mm}^{2}$ in c-plane and a thickness of $0.5-1 \mathrm{~mm}$. Opposing surfaces were polished to an optical grade in order to facilitate a homogenous strain distribution throughout the sample after application of uniaxial stress onto the corresponding c-plane using an apparatus similar to the system described in Ref. 7. The room temperature macro-Raman measurements were performed using a DILOR XY 800 triple grating Raman spectrometer with the $514.5 \mathrm{~nm}$ line of an $\mathrm{Ar}^{2+}$ laser as excitation source. All Raman spectra were recorded in backscattering geometry from an a-plane surface, where the

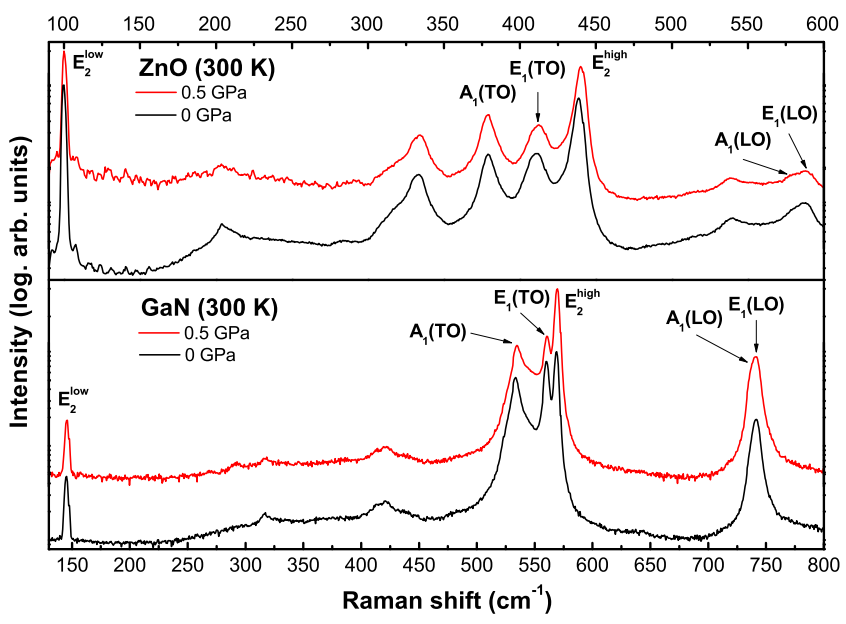

FIG. 1. (Color online) Raman spectra of $\mathrm{ZnO}$ and $\mathrm{GaN}$ for 0 and $0.5 \mathrm{GPa}$ uniaxial pressures parallel to the c-axis of the crystal. The spectra were vertically shifted for clarity. 


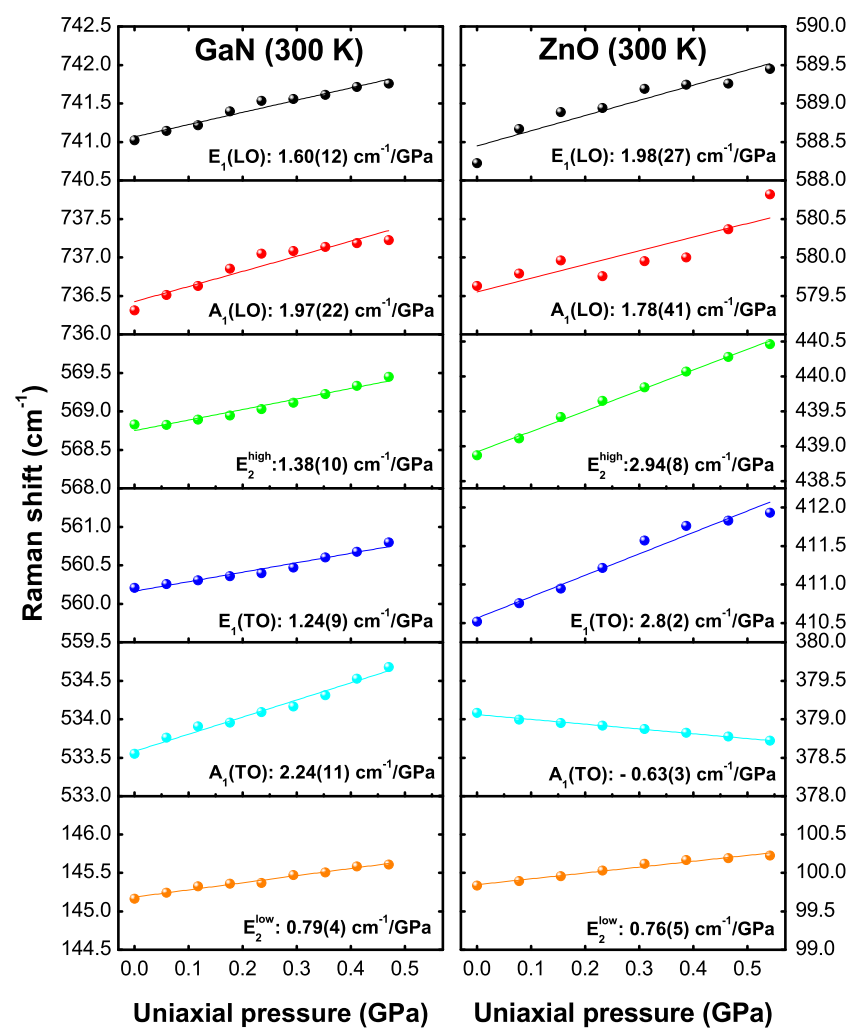

FIG. 2. (Color online) Uniaxial pressure parallel to the c-axis induced shifting behavior of all six first order phonon modes in $\mathrm{GaN}$ and $\mathrm{ZnO}$. Solid lines represent linear least-squares fits of the data points which result the stated errors in parentheses.

c-axis of the crystals was perpendicular to the direction of incidence of the laser light and parallel to the direction of the applied uniaxial stress $(\vec{c} \perp \vec{k}, \vec{c} \| \vec{P})$. The Raman spectra were collected with a liquid $\mathrm{N}_{2}$-cooled charge-coupled device array and calibrated with the spectral lines of a neon gas discharge lamp. All energies are uncorrected for the refractive index of air.

Figure 1 shows typical Raman spectra for $\mathrm{ZnO}$ and $\mathrm{GaN}$ at uniaxial pressures of 0 and $0.5 \mathrm{GPa}$. For the applied Raman geometry we clearly observe all the allowed optical first order modes $E_{2}^{\text {low }}, E_{2}^{\text {high }}, A_{1}(\mathrm{TO})$, and $E_{1}(\mathrm{TO})$ as well as the forbidden $A_{1}(\mathrm{LO})$ and $E_{1}(\mathrm{LO})$ modes $^{8}$ due to the large angular aperture of the optical system and crystal imperfections.

The resulting shifting behavior under the influence of uniaxial stress parallel to the c-axis for all phonon modes in $\mathrm{GaN}$ and $\mathrm{ZnO}$ is illustrated in Fig. 2 along with the corre- sponding linear least-squares fits to the data points. For almost all the phonon modes we observe a similar behavior with pressure, that is, as the pressure increases the modes shift to higher energies in accordance with theoretical predictions for $\mathrm{GaN},{ }^{9}$ except for $\mathrm{ZnO}$, where a negative pressure coefficient for the $A_{1}(\mathrm{TO})$ phonon mode is observed.

The pressure induced frequency shift $\Delta \omega$ for each phonon mode is given in Eq. (1) for the particular case of biaxially isotropic strain $\left(\epsilon_{x x}=\epsilon_{y y}\right)$ within the frame of Hooke's law,

$$
\begin{aligned}
& \Delta \omega=2 a \epsilon_{x x}+b \epsilon_{z z}=2 \tilde{a} \sigma_{x x}+\tilde{b} \sigma_{z z}, \\
& a=\widetilde{a}\left(C_{11}+C_{12}\right)+\widetilde{b} C_{13}, \quad b=2 \widetilde{a} C_{13}+\widetilde{b} C_{33} .
\end{aligned}
$$

Therefore, the measured value for $\Delta \omega$ provides the PDPs $a$ and $b$ for the case of known strain ( $\boldsymbol{\epsilon}$ ) or the phonon pressure coefficients $\widetilde{a}$ and $\tilde{b}$ for the case of given stress $(\boldsymbol{\sigma})$. The uniaxial pressure $\left(\sigma_{x x}=0\right.$ and $\left.\sigma_{z z} \neq 0\right)$ dependent Raman measurements directly yield $\tilde{b}$, whereas from hydrostatic pressure $\left(\sigma_{x x}=\sigma_{z z}\right)$ dependent Raman measurements we obtain $2 \widetilde{a}+\widetilde{b}$, which allows the determination of $\widetilde{a}$ and therefore also the determination of the PDPs $a$ and $b$ based on the linear system of equations from Eq. (2). The required elastic stiffness constants $C_{i j}$ for $\mathrm{GaN}$ and $\mathrm{ZnO}$ were taken from Brillouin scattering experiments. ${ }^{10,11}$ We remark that choice of a different set of $C_{i j}$ leads to a corresponding variation of the PDPs of up to $\approx 10 \%$.

Table I summarizes experimental and theoretical values for hydrostatic and uniaxial phonon pressure coefficients together with the resulting PDPs as calculated using Eq. (2). Comparison of the measured uniaxial phonon mode pressure coefficients of $\mathrm{GaN}$ with the theoretical values ${ }^{9}$ listed in Table I indicates fair agreement. The data also show that theory seems to underestimate uniaxial pressure coefficients as even recognizable for hydrostatic pressure dependent Raman measurements. ${ }^{5}$ In the uniaxial case for $\mathrm{GaN}$ the offset between the theoretical predictions and measured results decreases with increasing phonon energy, with the $E_{1}(\mathrm{LO})$ mode showing the only deviation from this trend. This observation suggests that it is not the sample quality which causes the observed offset but most likely an artifact which generally underestimates phonon mode pressure coefficients in the theoretical calculations. The uniaxial pressure values $\tilde{b}$ for GaN from Table I, as derived from Refs. 1 and 2, show a rather good agreement with the experimental values of this

TABLE I. Experimental and theoretical values for uniaxial $\widetilde{b}$ and hydrostatic $2 \widetilde{a}+\widetilde{b}$ pressure coefficients $\left(\mathrm{cm}^{-1} / \mathrm{GPa}\right)$ of Raman modes in GaN and $\mathrm{ZnO}$ along

\begin{tabular}{|c|c|c|c|c|c|c|c|c|c|c|}
\hline \multicolumn{7}{|c|}{$\mathrm{GaN}$} & \multicolumn{4}{|c|}{$\mathrm{ZnO}$} \\
\hline$A_{1}(\mathrm{TO})$ & $3.9(1)$ & 2.66 & 1.19 & $2.24(11)$ & $681(64)$ & $1067(73)$ & $4.91(5)$ & $-0.63(3)$ & $774(62)$ & $375(52)$ \\
\hline$E_{1}(\mathrm{TO})$ & $3.94(3)$ & 0.88 & 0.80 & $1.24(9)$ & $854(50)$ & $780(70)$ & $5.03(3)$ & $2.8(2)$ & $587(50)$ & $750(74)$ \\
\hline$E_{2}^{\text {high }}$ & $4.24(3)$ & $1.47 / 1.27(30)$ & 1.12 & $1.38(10)$ & $911(54)$ & $852(76)$ & $5.04(3)$ & $2.94(8)$ & $580(38)$ & $765(64)$ \\
\hline$A_{1}(\mathrm{LO})$ & $4.4(1)$ & $3.26(40)$ & 1.66 & $1.97(22)$ & $859(85)$ & $1042(111)$ & $4.56(7)$ & $1.78(41)$ & $577(80)$ & $599(99)$ \\
\hline Type/source & Expt. $^{a}$ & Expt. $^{\mathrm{b}}$ & Theor. $^{c}$ & & Expt. $^{\mathrm{d}}$ & & Expt. $^{\mathrm{e}}$ & & Expt. $^{\mathrm{d}}$ & \\
\hline
\end{tabular}
with the resulting phonon deformation potentials $\mathbf{a}$ and $\mathbf{b}$ in GPa. Numbers in parentheses are the errors. 


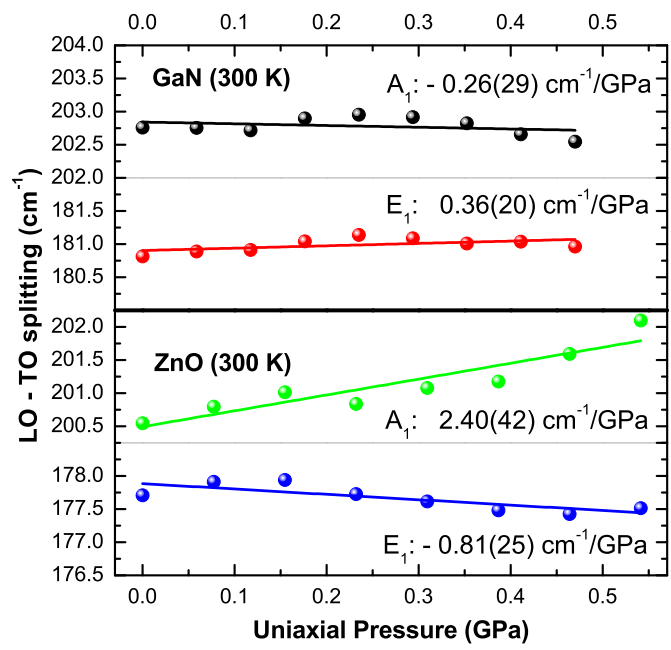

FIG. 3. (Color online) Uniaxial LO-TO-splitting pressure dependence $(\vec{p} \| \vec{c})$ of the $A_{1}$ and $E_{1}$ phonon modes in $\mathrm{GaN}$ and $\mathrm{ZnO}$. Solid lines represent linear least-squares fits of the data points which result the stated errors in parentheses.

work for the $E_{2}(h i g h)$ mode but significant deviations for the $E_{2}($ low $)$ mode. It should be noted that these indirectly derived values are usually obtained based on analyzing an inhomogeneously biaxially strained layer of GaN. Furthermore, the bulk Grüneisen parameters ${ }^{3}$ as a result of hydrostatic pressure dependent Raman measurement from a different sample type were utilized in order to determine the reported values. Table I also lists hydrostatic phonon mode pressure coefficients from Ref. 6 and the corresponding uniaxial results from this work for $\mathrm{ZnO}$. The combination of these values represents the special case that uniaxial and hydrostatic pressure dependent Raman measurements were performed with the same $\mathrm{ZnO}$ crystal, a Crystec $\mathrm{ZnO}$ substrate which originates from the same sample. This analysis assures equal zero pressure phonon line positions and facilitates direct derivation of PDPs in $\mathrm{ZnO}$.

The simultaneous observation of the LO and TO modes allows an accurate determination of the uniaxial pressure dependence of the LO-TO splittings in $\mathrm{ZnO}$ and $\mathrm{GaN}$ which are directly related to Born's transverse effective charge $e_{T}^{*}$ $\propto\left(\omega_{\mathrm{LO}}^{2}-\omega_{\mathrm{TO}}^{2}\right) .{ }^{12}$ For $\mathrm{ZnO}$ it is clearly shown in Fig. 3 that the LO-TO splitting of the $A_{1}$ phonon mode in $\mathrm{ZnO}$ increases in contrast to the splitting of the corresponding $E_{1}$ phonon mode which decreases with approximately a third of the rate. For GaN we observe a partly opposite influence of the uniaxial stress parallel to the c-axis on the LO-TO splitting of the $A_{1}$ and $E_{1}$ phonon modes. We measure, within the experimental error, a constant LO-TO splitting for the $A_{1}$ phonon mode of GaN and an increase of the LO-TO splitting for the $E_{1}$ phonon mode. Generally, it seems that the LO-TO splitting of the $A_{1}$ and $E_{1}$ modes in $\mathrm{ZnO}$ is more sensitive to the application of uniaxial stress than it is the case for GaN.

These observations can be understood using Kleinman's internal strain parameter $\zeta=(\alpha-\beta) /(\alpha+\beta)$ which correlates Keating's valence force field parameters $\alpha$ and $\beta$ for bond stretching and bond bending, respectively. Covalently bonded group IV materials such as silicon and carbon exhibit rather small values of this parameter, $\zeta \approx 0.3,{ }^{13}$ which is reflected by a strong hardening of their singlet and doublet phonon modes under the application of uniaxial stress parallel to the $[100]$-axis, ${ }^{7}$ for example. More ionically bonded
III-V materials such as $\mathrm{GaN}$ and GaAs exhibit larger internal strain parameters of $\approx 0.5$ and a comparatively weaker but still positive frequency shift of the LO/TO singlet and doublet modes. ${ }^{7,13,14}$ However, for II-VI materials such as cubic $\mathrm{ZnS}$ and wurtzite $\mathrm{CdS}$ a softening of the LO/TO singlet and the $A_{1}(\mathrm{TO})$ mode, respectively, has been observed ${ }^{15,16}$ which matches the result of this work for $\mathrm{ZnO}$ and is again accompanied by a rise of $\zeta$ to $\approx 0.7$. Consequently, the observed negative slope for the uniaxial pressure dependence of the $A_{1}$ (TO) mode in $\mathrm{ZnO}$ is the result of a larger bond ionicity and internal strain parameter when compared to GaN. Within this frame $\mathrm{GaN}$ deforms in a more affine manner in comparison to $\mathrm{ZnO}$ whose more ionic bonds exhibit a larger rigidity; i.e., the bonds of $\mathrm{ZnO}$ are more vulnerable to bond bending than to stretching. Therefore, it can be concluded that the observed partly opposing behavior for the uniaxial stress dependency of the LO-TO splittings of the $A_{1}$ and $E_{1}$ modes in $\mathrm{GaN}$ and $\mathrm{ZnO}$ is related to a difference in $\zeta$ and the accompanying difference in the bond charge distribution. Theoretical calculations would be desirable for a deeper understanding of the pressure dependent properties of the highly anisotropic bonds in II-VI materials.

In conclusion we provided uniaxial pressure coefficients and phonon deformation potentials for all first order zone center optical phonons in $\mathrm{GaN}$ and $\mathrm{ZnO}$ which facilitate the determination of strain levels by Raman spectroscopy. Comparison of the directly measured uniaxial Raman mode pressure coefficients with theoretical values from Wagner and Bechstedt ${ }^{9}$ indicates rather good agreement. Additionally, the influence of uniaxial stress parallel to the c-axis revealed an opposing behavior of the LO-TO splittings of the $A_{1}$ and $E_{1}$ modes in $\mathrm{ZnO}$ and $\mathrm{GaN}$ which yields information about the different ionicities of their bonds.

This work was supported by the DFG within Grant No. SFB787 and "UniCat" cluster of excellence.

${ }^{1}$ F. Demangeot, J. Frandon, M. A. Renucci, O. Briot, B. Gil, and R. L. Aulombard, Solid State Commun. 100, 207 (1996).

${ }^{2}$ V. Yu. Davydov, N. S. Averkiev, I. N. Goncharuk, D. K. Nelson, I. P. Nikitina, A. S. Polkovnikov, A. N. Smirnov, M. A. Jacobson, and O. K. Semchinova, J. Appl. Phys. 82, 5097 (1997).

${ }^{3}$ P. Perlin, C. Jauberthie-Carillon, J. P. Itie, A. San Miguel, I. Grzegory, and A. Polain, Phys. Rev. B 45, 83 (1992).

${ }^{4}$ M. R. Wagner, T. P. Bartel, R. Kirste, A. Hoffmann, J. Sann, S. LautenschlŠger, B. K. Meyer, and C. Kisielowski, Phys. Rev. B 79, 035307 (2009).

${ }^{5}$ A. R. Goñi, H. Siegle, K. Syassen, C. Thomsen, and J.-M. Wagner, Phys. Rev. B 64, 035205 (2001).

${ }^{6}$ J. S. Reparaz, L. R. Muniz, M. R. Wagner, A. R. Goñi, M. I. Alonso, A. Hoffmann, and B. K. Meyer, Appl. Phys. Lett. 96, 231906 (2010).

${ }^{7}$ M. Cardona, Phys. Status Solidi B 198, 5 (1996).

${ }^{8}$ T. C. Damen, S. P. S. Porto, and B. Tell, Phys. Rev. 142, 570 (1966).

${ }^{9}$ J.-M. Wagner and F. Bechstedt, Appl. Phys. Lett. 77, 346 (2000).

${ }^{10}$ A. Polian, M. Grimsditch, and I. Grzegory, J. Appl. Phys. 79, 3343 (1996).

${ }^{11}$ T. Azuhata, M. Takesada, T. Yagi, A. Shikanai, S. F. Chichibu, K. Torii, A. Nakamura, T. Sota, G. Cantwell, D. B. Eason, and C. W. Litton, J. Appl. Phys. 94, 968 (2003).

${ }^{12}$ P. Y. Yu and M. Cardona, Fundamentals of Semiconductors (Springer, Berlin, 2005), p. 303.

${ }^{13}$ R. M. Martin, Phys. Rev. B 1, 4005 (1970).

${ }^{14}$ K. Kim, W. R. L. Lambrecht, and B. Segall, Phys. Rev. B 50, 1502 (1994).

${ }^{15}$ M. Siakavellas, A. G. Kontos, and E. Anastassakis, J. Appl. Phys. 84, 517 (1998).

${ }^{16}$ R. J. Briggs and A. K. Ramdas, Phys. Rev. 13, 5518 (1976). 